\title{
Numerical Estimating the Shock Strength of Automobile Clutch Disc Hub
}

\author{
Cihat Gül \\ Mehmet Onur Genç \\ Ali Durmuş
}

Clutch disc hub is provide torque transmission between clutch disc drive plate and gearbox input shaft. Therefore disc hub is expected to show high endurance and storage under high torsional forces The aim of this paper is to investigate shock strength of a clutch disc hub numerically with finite element analysis. There are many engine and transmission elements in vehicle such as connecting rods, engine gears, bearing caps and clutch hubs need to have high resistance under high forces during driving conditions. In this study a clutch hub models shock strength simulation was obtained by finite element analysis with powder material and steel (will be produced by machining). Depending on the clutch hub geometry and materials such as powder materials and steel, the absorbed energies were calculated with finite element method and comparison between two materials were investigated.

Keywords - shock strength, clutch hub, charpy test, powder material

Cihat Gül

Valeo Automotive

Turkey

M.Onur Genç

Valeo Automotive

Turkey

Ali Durmuş / Associate Professor

Uludağ University

Turkey

\section{Introduction}

Clutch has the high importance in automobile and provides torque transmission in powertrain system. Flywheels are bolted to engine crankshaft and clutch cover assembly (3). During engagement, the disc (2) is clamped between the pressure plate and flywheel (1), resulting in torque flow from engine to transmission (Fig. 1).

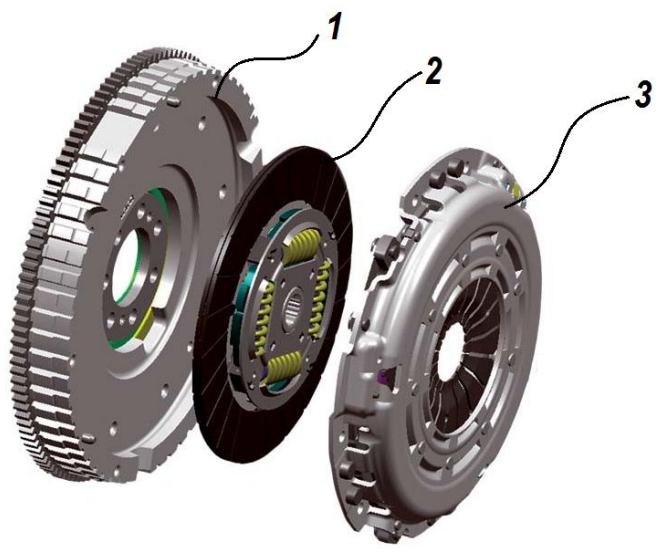

Figure 1. Clutch Systems

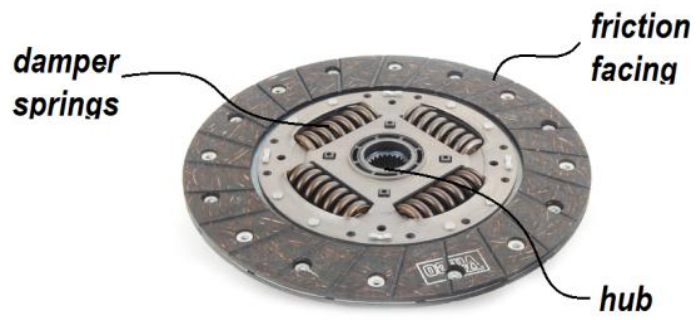

Figure 2. Clutch disc components (Valeo Automotive)

Clutch disc transmits clamped force through the friction from pressure plate and flywheel (Fig.2). During gear switching, drivers push the clutch pedal and clutch disc locates to disengagement position which provides torque cutting in order to gear change. Torque (T) which is transmitted by friction between flywheel and pressure plate is proportional with clutch disc friction coefficient $\mathrm{f}_{\mathrm{s}}$, clamp load $\mathrm{F}(\mathrm{N})$, number of friction surface $\mathrm{N}$ ve medium diameter of friction surface $R_{m}$ (mm).

$$
\mathrm{T}=\mathrm{f}_{\mathrm{s}} \cdot \mathrm{F} \cdot \mathrm{N} \cdot \mathrm{R}_{\mathrm{m}}
$$

Clutch disc hub is subjected to high rotational forces during driving conditions. Various driving conditions, especially abusive and unusual, lead to instant high stress occurrence on clutch disc hub with unstable torque transmission. This may cause breakage and crack on clutch disc hub which creates hazard for passengers and vehicle. Charpy test is of importance to evaluate the fragility endurance of materials. 


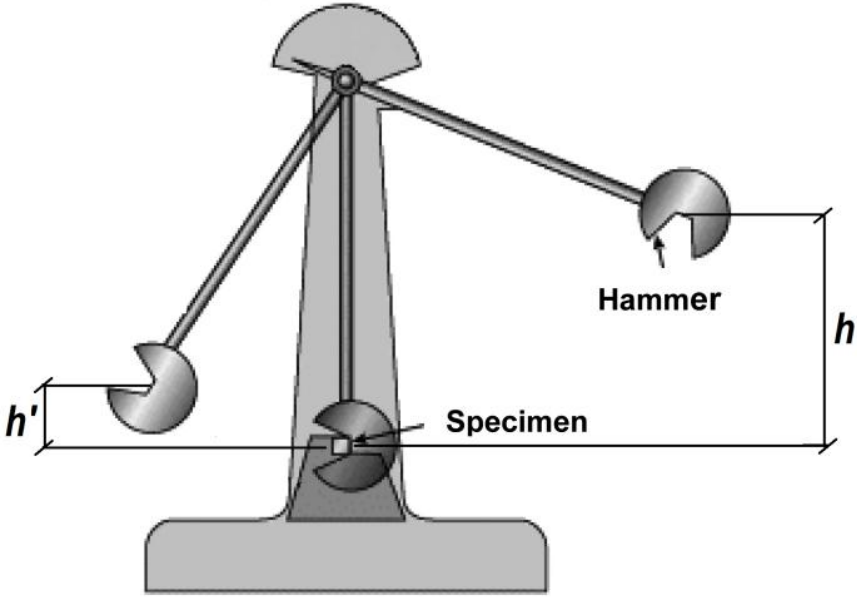

Figure 3. Charpy machine sketch

Ghaith (2010) invesigated in the study that the finite element modeling of Charpy impact test performed for a normalized carbon steel specimen based on plane strain geometry and bilinear isotropic hardening plasticity. It was found in that study a failure criterion is assumed to be at $10 \%$ of plastic strain based on the tensile experiment data and impact energy required for fracture of the selected steel specimen at room temperature (i.e. $25^{\circ} \mathrm{C}$ ) is to be 65.9 Joul [1]. Serizawa et al. (2001) investigated in their study that analyzing the dynamic crack growth in the Charpy impact test and a computer simulation method using the interface element sharp and round V-notch Charpy tests of steel was analyzing. In the case of the sharp V-notch Charpy test, the temperature effect appeared primarily through the surface energy of the crack while the influence of the yield stress ratio, representing the bulk property, was small [2]. Kumar et al. (2012) made a approach on the force time history obtained using an Instrumented impact test on steel at various impact velocities in the range 3.0 to $4.2 \mathrm{~m} / \mathrm{sec}$.The Second objective is to predict the stress concentration factor at the root of the V-notch in the test specimen using Finite Element Analysis. Finite Element Modelling is defined here as the analyst's choice of material models(constitutive laws), finite elements(of different types/shape/orders),meshes, constraints equations, analysis [3]. Emamian (2012) investigated in this study powder metallurgy technology that the results indicate that by appropriate selec-tion of process parameters, it is possible to obtain high wear resistance along with moderate toughness. By increasing the role of PM in industry which resulted from their ability to produce the complex shapes, high production rate, and dimension accuracy of final products, they need to be heat treated. Results of wear and hardness show considerable enhancement in mechanical properties of PM parts [4]. Zhu et al. (2015) investigated in this study the principle and method of drop-weight impact test, the impact resistance of concrete was measured using self-designed U-shape specimens and a newly designed drop-weight impact test apparatus. A series of drop-weight impact tests were carried out with four different masses of drop hammers $(0.875,0.8$,
0.675 and $0.5 \mathrm{~kg}$ ). The test results show that the impact resistance results fail to follow a normal distribution. In the study as expected, U-shaped specimens can predetermine the location of the cracks very well [5].

In this study numerical anlaysis was completed to clutch disc hub which is subjected to high rotational forces that causes instant shock on hub and leads to breakages and cracks. In the study two types of clutch disc hub material simulated with charpy test machine principles to see energy absorption property for both tested materials.

\section{Material and Method}

\section{A. Material}

Powder metallurgy is a term in which materials or components are made from metal powders. Powder metallurgy processes can greatly reduce metal removal processes, thereby reducing material losses in manufacture results in lower costs. Powder metallurgy components are used in a wide range of vehicle applications in automotive engines and transmissions due to their cost effectiveness as well as weight and energy savings. In the metal industry, resilience is an essential feature because it measures the brittleness of the material.

\section{B. Charpy Test Principles}

Resilience is one of the most important characteristic for material that using in vehicle body subjected to high forces. Clutch hub is directly exposed to immediate torque coming from engine that creates high forces.

As an impact blow from a weighted pendulum hammer that is released from a position at a fixed height $h$. The specimen is positioned at the base and with the release of pendulum, which has a knife edge, strikes and fractures the specimen at the notch. The pendulum continues its swing, rising a maximum height $h$ ' which should be lower than $h$ naturally. The energy absorbed at fracture E can be obtained by simply calculating the difference in potential energy of the pendulum before and after the test such as,

$$
\left.E=m . g .(h-h)^{\prime}\right)
$$

where $\mathrm{m}$ is the mass of pendulum and $\mathrm{g}$ is the gravitational acceleration.

In the FEA studies a velocity was defined as a boundary condition to the hammer and with no accelaration it was impacted to the hub ear. Velocity (V) of the hammer during at the impact was calculated with below formula:

$$
\mathrm{V}=2 \text {.g.h }
$$

"h" value used in the above formula isthe height of the hammer before test and was taken as $1.5 \mathrm{~m}$ and using the above formula velocity of the hammer during the impact was calculated as $5,4 \mathrm{~m} / \mathrm{s}$. 
Proc. of the Sixth International Conference on Advances in Mechanical and Robotics Engineering - AMRE 2017. Copyright $(\odot$ Institute of Research Engineers and Doctors. All rights reserved.

ISBN: 978-1-63248-140-5 doi: 10.15224/ 978-1-63248-140-5-37

\section{FEA Study}

Dynamic explicit finite element (FE) analysis of the Charpy impact test was conducted in this study to investigate the impact effect of the hammer on clutch disc hub ears. Two different material obtained by different production methods have been used and their material properties are as in table 1:

\begin{tabular}{|l|c|c|}
\hline & Steel - Machined Hub & Sintered Hub \\
\hline Density & $7,83 \mathrm{e}-9 \mathrm{Tonne} / \mathrm{mm}^{3}$ & $7,89 \mathrm{e}-9 \mathrm{Tonne} / \mathrm{mm}^{3}$ \\
\hline $\begin{array}{l}\text { Initial tensile } \\
\text { strength }\end{array}$ & $1195 \mathrm{MPa}$ & $742 \mathrm{MPa}$ \\
\hline
\end{tabular}

Table 1. Material Properties

Johnson-Cook material model was used for both material types. Investigation of the all the parameters of the JohnsonCook material model is ongoing and will be ended with a real test which will be the next step of this study.

As explained above velocity of the hammer was calculated and included to the analysis as a boundary condition with no acceleration. And hub was fixed from its inner teeths as in figure 4 . Schematic view of boundary conditions are as below:
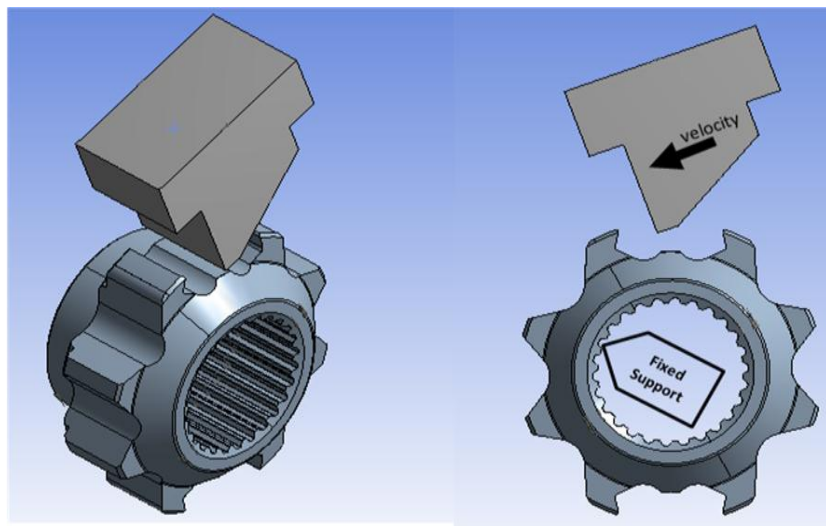

Figure 4. Schematic view of boundary conditions

\section{Analysis and Discussions}

At the end of the finite element analysis, absorbed energy amount have been obtained during the impact of the hammer to the clutch disc hub. Results and comparison of the materials can be summarized as below:

- Charpy test resutls can be simulated by Ansys Explicit Dynamics module if the target is to obtain the impact energy.
- As a next step simulation results has to be compared with a real test resulst and a correlation has to be developed.

- Absorbed energy levels of the hubs have gained by FEA as in figure 5.

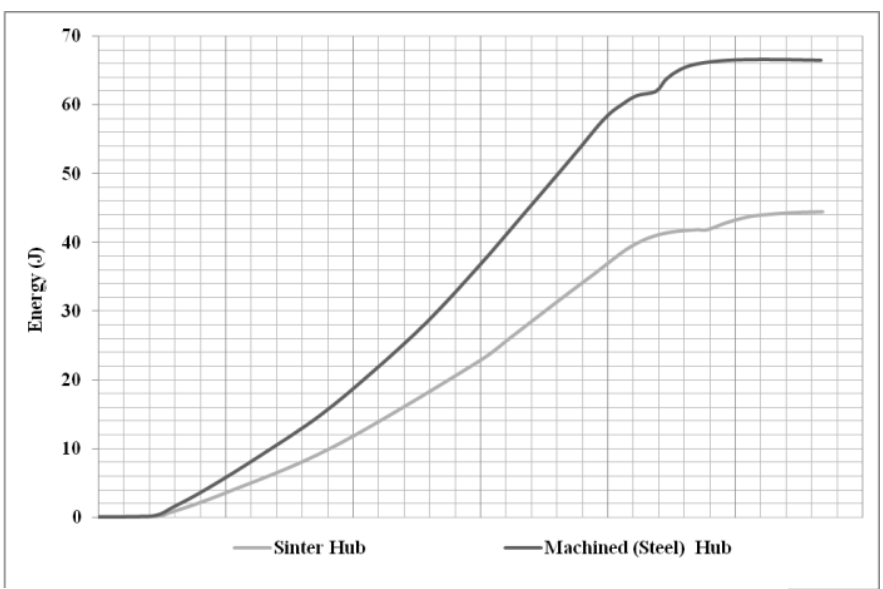

Figure 5. Absorbed energy during impact test

- Plastic strain occured during both analysis are at same level as seen on figure 6 .

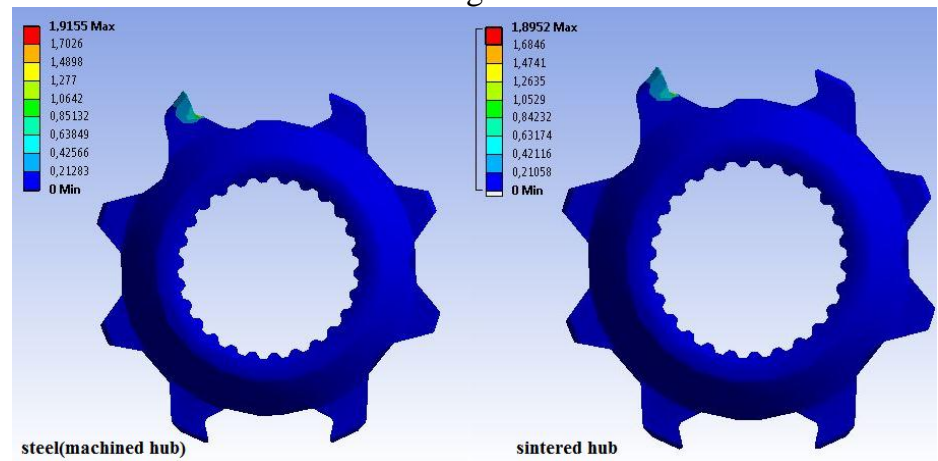

Figure 6. Plastic strain of the hubs during impact test

- Stress of the hubs during analysis reach their tensile strentgh value which means that during real test breakage will be start from the area have the most strain amount. Stress values can be seen from figure 7.

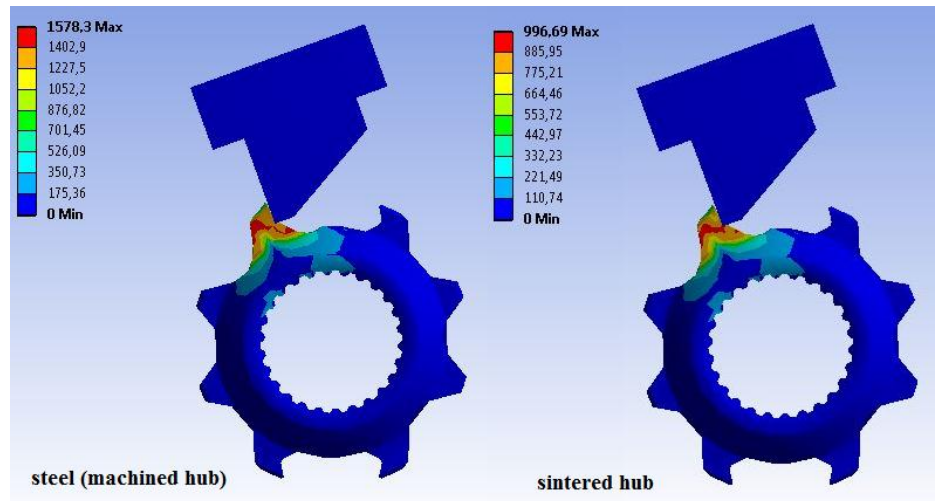

Figure 7. Stress of the hubs during impact test 


\section{Conclusion}

Powder materials are widely demanded recently especially in powertrain system components due to low cost and fast processing. Powder materials have some advantages and disadvantages compare to steel materials. One of the powertrain system component which can be produced by powder materials or steel (by machining) is clutch disc hub. Clutch disc hub is subjected to high dynamic forces in daily usage. Thereby, disc hub is expected to show high resistance to these forces under operational conditions. In this study the clutch disc hub geometry which was modeled by powder material and steel was compared with FEA. The charpy test which can measure and define the toughness of the material was modeled and performed to both materials. As a result and expected, steel show high resistance and absorbed more enegry than the powder materials which produced by sintering. In the further studies, the clutch disc hub, which was modeled and analyzed in this study, will be produced with steel and powder material and comparative charpy test will be performed to both hub.

At the end of the studies a FEA procedure with correlated material model for sinter materials will be gained and for the future studies instead of a real test, validation of the hub designs will be fixed with FEA calculations only.

\section{References}

[1] F. A. Ghaith, " Nonlinear Finite Element Modeling of Charpy Impact Test.' Advanced Materials Research, Vols. 83- 86, pp 182-189, 2010

[2] H. Serizawa, Z. Wu, H. Murakawa, ' Computational Analysis of Charpy Impact Tests Using Interface Elements." Transactions of JWRI, Vol. 30, pp 97-102, 2001

[3] K. M. Kumar, M.R. Devaraj, H.V. LakshmiNarayana, 'Finite Element Modelling for Numerical Simulation of Charpy Impact Test on Materials." International Conference on Challenges and Opportunities in Mechanical Engineering, Industrial Engineering and Management Studies, pp 32-36, 11-13 July, 2012.

[4] A. Emamian, " A Study on Wear Resistance, Hardness and Impact Behaviour of Carburized Fe-Based Powder Metallurgy Parts for Automotive Applications." Materials Sciences and Applications, Vol.3, pp 519-522, 2012.

[5] X. Zhu, H. Zhu, H. Li, " 5,4Drop-Weight Impact Test on U-Shape Concrete Specimens with Statistical and Regression Analyses.' Journal of Materials, Vol. 8, pp 5877-5890, 2015.

[6] T.Lorrit, "'Specimen loading detemined by displacement measurement in instrumented charpy impact test." Engineering Fracture Mechanics, Vol.65 (2000), pp. 703-713.

[7] N. Jassim, A. J. Farhan, R. Hilal ' Charpy Impact TestFor SiO2 (NanoMicro) Particles/Epoxy Composites.' International Journal of Recent Scientific Research, Vol. 6, Issue, 6, pp.4623-4628, June, 2015

[8] M. Bruchhausen, S. Holmström, I. Simonovski, T. Austin, J.-M. Lapetite, S. Ripplinger, F. de Haan, " Recent developments in small punch testing: Tensile properties and DBTT." Theoretical and Applied Fracture Mechanics, Vol. 86, pp 2-10, 2016.

[9] N. D. Alexopoulos, "Impact properties of the aircraft cast aluminium alloy Al-7Si- 0.6Mg (A357).' EPJ Web of Conferences 6, 02002, 2010.

[10] N. Ohtsuka, Y. Shindo, A. Makita, 'Evaluation of hydrogen embrittlement and temper embrittlement by key curve method in instrumented Charpy test' EPJ Web of Conferences 6, 14004, 2010. 